18

\title{
Which way to KIC?
}

\author{
David C. Brown
}

AI in Design Group,

Worcester Polytechnic Institute,

Department of Computer Science,

100 Institute Rd., Worcester, MA 01609

Ph: (508) 831-5618; FAX: (508) 831-5776

Email:dcb@cs.wpi.edu

\begin{abstract}
This paper is a report of the discussions of three working groups at the KIC-2 workshop. The groups were assigned to discuss System Architectures, Representations, and Delivered Systems, and were charged with addressing topics from the point of view of Research Achievements, Research Issues, and Industry Needs.
\end{abstract}

\section{Keywords}

System Architectures, Representations, Delivered Systems.

\section{KIC-OFF}

This paper is a report of the points raised and the conclusions reached by three working groups that were formed from the attendees at the KIC-2 workshop. The groups were assigned to discuss System Architectures, Representations, and Delivered Systems. Each group was charged with thinking about their topic from the point of view of Research Achievements, Research Issues, and Industry Needs.

Much of this paper will be based on the deliberations of the Architectures group, as this was the group of which the author was a member, and because it was observed that most of the points raised there were also discussed by the other groups. The issues from all three groups will be summarized in this paper.

In order to characterize the achievements of the KIC field we will focus on the state of research ten years ago, and then contrast that with current research. The difference should reveal the Research Achievements, i.e., which problems the field thinks are solved. The description of current research will also reveal the Research Issues, as this is what people are currently focussing on. Surprisingly, at first anyway, it also reveals the Industry Needs. 


\section{THE EARLY GAME}

By 1986, ten years ago at the time of writing, IFIP had already had two Working Conferences about knowledge-based CAD [Latombe 1978] [Gero 1985].

That early research was characterized by attempts to tackle small design problems in a single domain (e.g., mechanical), from the point of view of a single designer. Research was mostly concerned with the detailed, parametric design of single components and simple assemblies, usually using routine design problemsolving.

Many systems were rule-based, although there were some attempts, such as my own [Brown \& Chandrasekaran 1985], that started to break away from general purpose reasoning to characterize the knowledge and problem-solving needed for design and configuration problems. Most systems were autonomous, responding to design requirements with no human in the loop. $\mathrm{CAD}$ systems were mostly wire frame, with some solid modelling capabilities starting to appear.

\section{ACHIEVEMENTS \& RESEARCH ISSUES}

In the last 10 years, research has progressed quite far beyond the situation in 1986. Research is now concerned with large designs in multiple domains (e.g., mechanical plus electrical), and the large systems that design them. These systems often incorporate "legacy" systems, and treat design as part of the enterprise - using an open architecture, integrating with business systems, and involving physical distribution.

The reasoning involved in these design systems is often quite mixed, due to the incorporation of real or simulated teams where members have different points of view (representing different lifecycle issues, for example), as well as the explicit incorporation of multiple types of reasoning (e.g., requirements specification and checking, abstraction, problem decomposition, functional reasoning, etc.).

Many systems concentrate on early design, especially given that a large amount of the product's cost can be locked in at the conceptual design stage. In addition, as routine design is quite well understood, most research now tackles non-routine design.

CAD systems are now parametric, feature based, include attributes and constraint checking. Some even include small amounts of intelligent reasoning. Achievements in the areas of geometric reasoning and representations have been quite strong. Research into features is very active, and their definition, their relationship to function, their role in reflecting the designer's intent, and their dependence on viewpoint (e.g., manufacturing vs. assembly) are topics that produce much disagreement and discussion - and this workshop was no exception.

There is recognition that designs need to be represented in more than one way, at different levels of abstraction, from different viewpoints. This might include product-process relations. A key research issue is the need for links across these 
heterogeneous representations, possibly between modelers, to connect related information and data.

Embedded knowledge-based reasoning has become quite common, and small expert systems are quite widely used, and have had a noticeable impact on the design community. Some effort is being given to the design of intelligent artifacts, that incorporate knowledge-based action, and knowledge about the artifact itself (e.g., for reasoning about diagnosis or repair).

In addition, there has been recognition of the importance of:

- the need for design rationale (design intent) capture and use;

- the fact that both design knowledge and design data is changing over time;

- the increased use of design-related data bases, brought about by larger designs, design reuse, and the use of legacy systems (that may have associated data bases);

- the fact that design is often a discussion between people, with appropriate support required;

- the need to recognize inconsistency in designs and design information;

- the study of and comparison between design methodology across domains.

This set of changes in research over ten years points to many achievements that have allowed the field to progress to these new topics. For example, routine parametric design is considered to be a solved problem, and CAD systems are more properly thought of as solid modelers.

This set of changes also indicates most of the current research topics. The move to bigger design problems, less-routine activity, a stress on earlier design stages, with large amounts of varied knowledge raises many research issues. The move towards distributed design and the integration of design systems into the existing business structure raises' many more.

\section{OUR GOAL}

Research into design representation, reasoning and systems can, quite properly, have the goal of better understanding all aspects of design. However, design is such a practical area, that we need to have "satisfying the needs of industry" as one of our goals.

The basic needs of industry - making products better, cheaper, and faster haven't changed much in ten years. However, there is now a recognition that the design process has an architecture, that can be examined and refined. Industry needs tools which match this architecture that can support both the design process and the individuals involved in it. The design process needs to be integrated into the enterprise. In addition, the design process is seen as something that can and should be 'instrumented' to assist with the management of quality.

Industry is becoming increasingly aware that knowledge is a key resource that is important to capture, keep and use. There's a need for design rationale capture to 
allow explanations to be provided about existing designs, to guide new designs, and to allow easier redesign activities.

Systems with embedded intelligence are no longer strange - although work explicitly labelled as Artificial Intelligence may still be treated with suspicion.

Even though industry's basic needs haven't changed much, business organizations and practices are changing, with more distribution of design teams and designs. Virtual prototyping has reduced the number of actual prototypes being made. There is increased use of existing parts, via catalogs, and existing systems (i.e., legacy systems), in order to maximize return on investment. Rapid prototyping (real, not virtual) needs to be more strongly tied to design systems. Such needs require open systems to ensure maximum flexibility.

Design in industry is increasingly concerned with life cycle issues and impact (for example, as in "green engineering"). Design for manufacturability is more common now, and new "ilities" are appearing - it has been claimed that "the number of "ilities are growing".

It should be clear that these observations about "industry needs", include many of current research issues that were discussed above. In addition, the systems delivered have addressed some of these issues, with better integration via data exchange, increased use of features and product models, wide support for detailed design, and growing concern for supporting conceptual design. One explanation for this is that design researchers have been listening to industry, and vice versa. Clearly, this needs to continue.

In summary, industry needs distributed, integrated, design systems. There is a strong correlation between industry needs and current design research.

\section{AFTER LOTS OF KIC-ING, WHAT'S THE SCORE?}

Ten years ago we were concerned with "IntCAD", with intelligence in the CAD system. Now we are focussing on "KIC", Knowledge Intensive CAD. KIC has knowledge of many types in many places, is concerned with product knowledge management, and its relation to lifecycle stages.

\section{REFERENCES}

D.C. Brown \& B.Chandrasekaran (1985) Expert Systems for a Class of Mechanical Design Activity, Knowledge Engineering in Computer-Aided Design, J.S.Gero (Ed.) North Holland, pp. 259-282.

J.S. Gero (1985) Knowledge Engineering in Computer-Aided Design. North-Holland, Amsterdam.

J-C. Latombe (1978) Artificial Intelligence and Pattern Recognition in Computer Aided Design. North-Holland, Amsterdam. 


\section{BIOGRAPHY}

David C. Brown is a Professor of Computer Science at WPI, and is a member of the ACM, IEEE Computer Society, AAAI, and IFIP WG5.2. He is on the editorial boards of the Journals AI in Engineering, Design, Analysis and Manufacturing and Concurrent Engineering: Research and Applications. His research interests include computational models of Engineering Design, and the applications of Artificial Intelligence to Engineering. He is the author, with B. Chandrasekaran, of the book Design Problem Solving: Knowledge Structures and Control Strategies, Pitman Publishing, Ltd., and a co-editor of Intelligent Computer-Aided Design, Elsevier Science Publishers B.V. (North-Holland). 